\title{
Form symbolism, analogy, and metaphor
}

\author{
CHANG HONG LIU and JOHN M. KENNEDY \\ University of Toronto, Scarborough, Ontario, Canada
}

\begin{abstract}
Simple forms, such as a square and a circle, can be symbolic; for example, a square can be deemed to be hard and a circle to be soft. The relation between form symbolism and the comprehension of metaphors and analogies was studied in three experiments. Subjects were asked to rate matches between terms such as soft and hard and circle and square as symbols (Experiment 1), metaphors (Experiment 2), and analogies (Experiment 3). The results show that a highly rated symbolic relation could be a poorly rated metaphorical relation. Ratings of analogies were similar to ratings of symbols. We argue that apt metaphors, analogies, and symbolic forms claim that the vehicle and the topic of the comparisons have common features, but that metaphoric representation entails more common features than does either symbolism or analogy, because metaphor requires that the vehicle be an especially apt example of a superordinate class. Thus, metaphor is a particularly strong claim about common features shared by the topic and the vehicle.
\end{abstract}

A circle can symbolize an abstract idea, such as the universe or contentment. Here, we use the phrase form symbolism for connections made between abstract referents and forms such as straight and curved lines (Liu \& Kennedy, 1993, 1994, 1996; Marks, 1996). Liu and Kennedy point out that, if subjects are asked to match circle and square with warm and cold or with weak and strong, subjects match the circle with warm and weak and the square with cold and strong. We may conventionally say, "he is a square." However, Liu (1997) argues that convention is not the sole basis for form symbolism, for subjects use sphere and cube much as they use circle and square, whereas curved and angular generated the same consensus as curved and straight. Liu notes that square and straight share many more conventional uses than do square and cube. He concludes that the form symbolism tasks employ common features of forms (spheres and circles have many common features, as do cubes and squares) rather than just implications from idioms (in which square shares referents with straight).

Some take form symbolism to be an ellipsis for a metaphor-that is, a form can be used in a metaphor for a referent if it can be a symbol for the referent (Gombrich, 1963, 1965; Hausman, 1989; Indurhkya, 1992; Johnson, 1991). This could be called the "metaphor-identity view." But, we suggest here, symbolism may be closer to analogy than to metaphor.

Liu and Kennedy (1996) argue that metaphors use class-inclusion-that is, putting topics into higher order classes (Glucksberg \& Keysar, 1990; Richards, 1936), much as we do in "a dog is an animal." In this case,

We are greatly indebted to J. Vervaeke, C. D. Green, and A. Katz for their useful suggestions on the designs of the experiments. We also thank J. Seemangul for helping to conduct one of the experiments. Correspondence should be addressed to C. H. Liu, Department of Psychology, McGill University, 1205 Dr. Penfield Avenue, Montreal, PQ, Canada H3A 1B1 (e-mail: chang@ego.psych.mcgill.ca). the lower order term $(\operatorname{dog})$ precedes the higher order term (animal). In "surgeons are butchers," the vehicle term butchers is taken as indicating a higher order classnamely, the relatively crude. The tenor term surgeons is put in the higher order class indicated by the vehicle (Glucksberg \& Keysar, 1990; Kennedy, 1990, 1993; Kennedy, Green, \& Vervaeke, 1993). Butchers are a prime example of their class. Symbols, on the other hand, refer to something, without claiming that the referent is an instance of the symbol's class. When a circle is used as a symbol for mother, it is a label or name referring to mother, not a prime example of a superordinate category for mother.

Prime examples have many of the defining features of the class. As Glucksberg and Keysar (1990) say, "He is a Bela Lugosi" means that he has all the characteristics Lugosi exemplifies. Do form symbols entail many implications about relevant features? If not, they might succeed as symbols but fail when turned into metaphors.

Tourangeau and Sternberg $(1981,1982)$ propose that metaphors map two distant domains (e.g., items from "between-domains" include a world leader and a lion) using close "within-domain" similarities (such as aggressiveness and prestige). In this view, both metaphors and analogies compare two systems of relations. "Richard is a lion" means "his status among his peers is the lion's status among animals." If this is the case, and if the metaphor-identity view is correct about form symbols, then form symbols could be the basis for analogies as well as for metaphors. For Gentner and Jeziorski (1993), an analogy is "a mapping of knowledge from one domain (the base) into another (the target) such that a system of relations that holds among the base objects also holds among the target objects" (p. 449). The definition is akin to Tourangeau and Sternberg's account of metaphor. However, an analogy may stress only a few features and may not require the vehicle of the analogy to be an especially prototypical example of its type. 
Table 1

Percent Agreement of Words With Shapes, Derived From Matchings in Liu and Kennedy (1993)

\begin{tabular}{llr}
\hline Circle & Square & \% Agreement \\
\hline Soft & Hard & 100 \\
Happy & Sad & 94 \\
Mother & Father & 94 \\
Love & Hate & 89 \\
Good & Evil & 89 \\
Bright & Dark & 87 \\
Alive & Dead & 87 \\
Light & Heavy & 85 \\
Summer & Winter & 81 \\
Warm & Cold & 81 \\
Fast & Slow & 79 \\
Weak & Strong & 79 \\
Spring & Fall & 74 \\
Cat & Dog & 74 \\
Quiet & Loud & 62 \\
Walking & Standing & 62 \\
Even & Odd & 57 \\
Animal & Plant & 53 \\
Far & Near & 53 \\
Deep & Shallow & 51 \\
\hline
\end{tabular}

Note-The values in the table represent the percentage of occasions on which the first word of each pair was matched with the circle and the second word of the pair was matched with the square.

Perhaps a straight line means quiet insofar as a crooked line means noisy, with a system of shapes mapping onto a system of noises. Being noisy involves many sounds and a crooked line involves many bends, whereas a straight line and a quiet period have in common a lack of change (Leach, 1976).

Consider a familiar example - a solar system analogy for the atom (the topic of the analogy, in the terms we are using). In this analogy, the electron is to the nucleus as the earth is to the sun. Interestingly, despite the analogy being well known, "the earth is an electron," stated on its own, is not readily understood-it is forced at best. That is, although $\mathrm{X}: \mathrm{Y}:: \mathrm{A}: \mathrm{B}$ is acceptable, a part of this analogy-X : A - is suspect as a metaphor on its own. But the theory that metaphors are elliptical analogies, taken literally and straightforwardly, not only claims that one can unpack a metaphor to find an analogy, but also that one can take a familiar analogy and readily extract a metaphor from it. However, if metaphors are "stronger" claims than analogies, it would not follow that a metaphor could be extracted from an acceptable analogy.

Symbols, metaphors, and analogies are examined here in three rating experiments. The stimulus materials are from an earlier "matching" study (Liu \& Kennedy, 1994). In it, subjects matched 20 word-pairs-antonyms or contrasts, such as mother and father - -with 2 shapes - a circle and a square. Table 1 shows the levels of consensus found. The results revealed a significant consensus, ranging from $100 \%$ (soft-hard) to chance level $(51 \%$ on deep-shallow). Levels above $66 \%$ are above chance $(\mathrm{CR}=2.04, p<.05)$. When subjects agree that certain meanings fit well with a shape, does it follow that the shape can be used in metaphors that have those meanings? Or in "analogies"? To find out, we used the 20 word-pairs in Table 1 and the words circle and square to compose symbols (Experiment 1), metaphors (Experiment 2), and analogies (Experiment 3). Subjects were asked to rate the symbols, metaphors, or analogies as good or poor.

\section{EXPERIMENT 1 Rating the Shapes as Symbols}

The forms produce a high level of consensus as symbols, Table 1 shows. But to say a word fits better with one shape than the other does not indicate the degree of the fit. This ambiguity can be resolved by using a rating method to study symbolism, taking each word on its own.

\begin{abstract}
Method
Subjects. Forty-two undergraduates (ranging from 19 to 42 years of age, with a median age of 21,31 of whom were females) from an introductory psychology course participated and were tested individually.

Materials and Procedure. Subjects were given sentences of the form "An X is a good symbol for Y." X was replaced by the words circle or square and $\mathrm{Y}$ replaced by the words shown in Table 1. (The wording " $\mathrm{X}$ is a good symbol for ..." was chosen because " $\mathrm{X}$ is a symbol for ..." might imply only that $\mathrm{X}$ is a familiar symbol for the referent.) Every word was used in two sentences-one with circle, the other with square - for a total of 80 sentences. In order to fit the grammar of the sentence, the adjectives shown in Table 1 were transformed into their noun forms. Thus, the word soft was changed to softness in the sentence "A circle is a good symbol for softness." Likewise, happy and sad became happiness and sadness, and so on. Any ambiguity in the case of spring and fall was resolved by adding the phrase the season in parentheses after the sentence. The scale was from 0 (poor) to 5 for a good symbol. Each subject rated 80 sentences, in different order in each case. The examples in the instructions involved the symbolic use of a triangle, as follows: "A triangle, for example, was used as a symbol for Godhead in ancient Egypt, a symbol of wisdom in ancient Greece, and a symbol of creativity and energy in ancient India." Sentences following consensus in Table 1 can be called congruent sentences, and the reverse incongruent sentences.
\end{abstract}

\section{Results and Discussion}

Mean ratings for each word-pair are shown in Table 2. A $2 \times 2$ repeated measures analysis of variance (ANOVA) applied to the means - with circle-word and square-word as word factor and circle and square as shape factorfound a main effect of shape, with words paired with circle rated higher $\left[F(1,41)=34.00, M S_{\mathrm{e}}=0.14, p<.0001\right]$ and a significant interaction between word and shape factors $\left[F(1,41)=179.67, M S_{\mathrm{e}}=0.30, p<.0001\right]$. The word factor was nonsignificant.

Comparisons of means $(\alpha=.05)$ are shown in Table 2. Most significant differences attach to high consensus words. Also, significant differences are found in at least one of the words from the top $10 \mathrm{high}$-consensus pairs (i.e., 19 out of 20 words). The exception is the word winter. There are five cases (all in the five lowest consensus pairs, none of which was significantly higher than chance in Table 1) where the mean ratings of words fitted with circle in Table 1 were higher when they were paired with square or where the mean ratings of square-words were higher when they were paired with circle. It is striking that of the five, only one (even) reached significance.

Correlation analyses found positive correlations between the ratings of circle-words paired with circle here 
Table 2

Symbol (Experiment 1) and Metaphor (Experiment 2) Ratings

\begin{tabular}{|c|c|c|c|c|c|c|c|c|c|}
\hline \multirow[b]{2}{*}{ Word } & \multicolumn{2}{|c|}{ Symbol } & \multicolumn{2}{|c|}{ Metaphor } & \multirow[b]{2}{*}{ Word } & \multicolumn{2}{|c|}{ Symbol } & \multicolumn{2}{|c|}{ Metaphor } \\
\hline & Circle & Square & Circle & Square & & Circle & Square & Circle & Square \\
\hline Softness & $3.62^{*}$ & 0.74 & $1.11^{*}$ & 0.14 & Hardness & $1.07^{*}$ & 4.10 & $0.39^{*}$ & 0.92 \\
\hline Happiness & $4.07^{*}$ & 1.52 & $1.86^{*}$ & 0.14 & Sadness & $1.69^{*}$ & 2.43 & $1.39^{*}+$ & 0.58 \\
\hline Mother & $3.69^{*}$ & 0.93 & $0.94^{\dagger}$ & 1.06 & Father & $1.83^{*}$ & 3.12 & $0.67^{*}$ & 1.61 \\
\hline Love & $4.10^{*}$ & 0.95 & $3.11^{*}$ & 0.56 & Hate & $1.17^{*}$ & 2.88 & $2.17^{* \dagger}$ & 0.69 \\
\hline Goodness & $3.83^{*}$ & 1.62 & $1.42^{*}$ & 0.17 & Evil & $1.31^{*}$ & 2.81 & $1.67^{* \dagger}$ & 0.67 \\
\hline Brightness & $3.79^{*}$ & 1.36 & $0.81^{*}$ & 0.19 & Darkness & $1.93^{*}$ & 2.90 & $0.72^{\dagger}$ & 0.33 \\
\hline Life & $4.55^{*}$ & 1.67 & $3.17^{*}$ & 1.19 & Death & $1.86^{*}$ & 2.76 & $1.17^{\dagger}$ & 0.92 \\
\hline Lightness & $3.76^{*}$ & 1.55 & 0.61 & 0.14 & Heaviness & $1.83^{*}$ & 3.50 & $0.58^{\dagger}$ & 0.56 \\
\hline Summer & $3.33^{*}$ & 1.60 & $0.86^{*}$ & 0.22 & Winter & 2.31 & 2.64 & $1.22^{* \dagger}$ & 0.56 \\
\hline Warmth & $3.71^{*}$ & 1.43 & $1.36^{*}$ & 0.11 & Coldness & $2.14^{*}$ & 3.00 & 0.42 & 0.47 \\
\hline High speed & $2.67^{*}$ & 1.76 & 0.86 & 0.22 & Low speed & 2.00 & 2.19 & 0.53 & 0.67 \\
\hline Weakness & $2.29^{*}$ & 1.40 & $0.83^{*}$ & 0.28 & Strength & $2.50^{*}$ & 3.57 & $1.19^{\dagger}$ & 0.92 \\
\hline Spring & $2.95^{*}$ & 1.55 & $1.08^{*}$ & 0.44 & Fall & 2.05 & 2.43 & $0.86^{\dagger}$ & 0.53 \\
\hline Cat & $2.45^{*}$ & 1.02 & 0.44 & 0.22 & Dog & 1.57 & 1.90 & $0.44^{\dagger}$ & 0.39 \\
\hline Quietness & $3.19^{*}$ & 1.98 & $0.89^{*}$ & 0.19 & Loudness & 2.67 & 2.81 & $0.58^{\dagger}$ & 0.31 \\
\hline Walking & $1.95^{+}$ & 2.19 & $1.14^{*}$ & 0.42 & Standing & $1.57^{*}$ & 2.62 & 0.39 & 0.53 \\
\hline Evenness & $3.00^{*+}$ & 3.69 & 1.00 & 1.14 & Oddness & $1.40^{*}$ & 2.52 & $0.39^{*}$ & 1.42 \\
\hline Animal & 2.12 & 1.52 & 0.58 & 0.25 & Plant & $2.211^{\dagger}$ & 1.79 & 0.47 & 0.25 \\
\hline Distance & $2.00^{*+}$ & 2.60 & 0.72 & 0.44 & Nearness & $2.67^{* \dagger}$ & 1.90 & 0.31 & 0.33 \\
\hline Deep & 2.79 & 2.64 & 0.44 & 0.22 & Shallow & 1.86 & 2.38 & $0.61^{\dagger}$ & 0.22 \\
\hline Means & 3.19 & 1.69 & 1.17 & 0.39 & Means & 0.80 & 1.87 & 2.72 & 0.63 \\
\hline
\end{tabular}

* Significant difference between the two ratings of the same word, within Experiment 1 or within Experiment 2.

${ }^{\dagger}$ The values of the two means are in the opposite direction to consensus in Table 1.

and the levels of consensus in Table $1(r=.78, p<.0001)$, and likewise for square-words $(r=.48, p<.05)$. Furthermore, negative correlations are found between circlewords paired with square and the consensus $(r=-.83$, $p<.0001$ ) and between square-words paired with circle and the consensus $(r=-.59, p<.01)$.

Circle was generally considered a better symbol than square. The grand means for circle and square were 3.19 and 2.72 , respectively. In part, we attribute this difference to the salience and variety of the properties of a circle (very smooth, especially symmetrical, etc.) and in part we attribute it to the choice of terms on which comparisons were based in this experiment. We suggest that, with a selection of suitable referents where straightness, roughness, sharpness, and so forth, were relevant, the square would achieve higher ratings. This is in the nature of slight biases in the selection of stimuli for experiments on representation, comparisons, tropes, and so forth, and is not our focus here, inasmuch as the stimuli used in each experiment originated from the same words (listed in Table 1).

Results from symbol rating were highly consistent with the consensus in Table 1. But can the pairings of words and shapes also function as metaphors?

\section{EXPERIMENT 2 \\ Rating the Shapes as Metaphors}

Metaphors have the form X is a Y. Often, the metaphor's presence is signaled by using an emphatic qualifier (e.g., real) as in "His job is a real jail." In Experiment 2, we used real as a qualifier in place of the emphatic word good employed in Experiment 1, inasmuch as metaphors do not generally use good as an emphatic qualifier, thereby avoiding oxymorons such as "the man is a good shark." Some of the metaphors produced from Table 1's examples by this method are clichés_- "father is a real square," "life is a real circle," and "love is a real circle." These clichés can be helpful as a check on the procedure. If the nonclichés are rated at the same level as the clichés, then they are functioning at a level comparable to that of familiar metaphors.

\section{Method}

Subjects. Thirty-six undergraduates (ranging from 21 to 47 years of age, with a median age of 22,22 of whom were females) from an introductory psychology course participated and were tested individually.

Materials and Procedure. The questionnaire for Experiment 2 was as in Experiment 1 except the sentence form was changed from " $X$ is a good symbol for $Y$ " to " $\mathrm{X}$ is a real $\mathrm{Y}$." Examples of metaphors used in the instructions were "The soldier is a real hawk," "She is a real dragon," and "His job is a real jail."

\section{Results and Discussion}

The means for most of the sentences are below one, and many of the rest are below two (Table 2). The two exceptions are the clichés, "Love is a real circle" and "Life is a real circle," which have a mean rating of 3.11 and 3.17 , respectively. Another cliché - "Father is a real square"was the highest rated sentence using a square, at a rating of 1.61. The responses evidently reflect knowledge of clichéd uses of shapes, but this knowledge did not lead to many strong ratings for metaphoric uses of the shapes.

Four important comparisons can be made between the metaphor ratings in this experiment and the symbol ratings in Experiment 1. First, the mean ratings for the symbols are higher than the mean ratings for the metaphors 
(77 out of $80, z=8.16, p<.0001$ ). Second, in metaphor rating, $47.5 \%$ of the multiple mean comparisons are found to be significant, whereas in symbol rating, $75 \%$ of the multiple comparisons are found to be significant $\left[\chi^{2}(1)=6.37, p<.02\right]$. Third, more mean values are in the opposite direction to consensus in the metaphor rating table $(32.5 \%)$ than they are in the symbol rating table $(12.5 \%)\left[\chi^{2}(1)=4.59, p<.05\right]$. Last, negative correlations between ratings of the circle-words paired with square and the levels of consensus and between ratings of square-words paired with circle and levels of consensus are found in the symbol-rating experiment but not in the metaphor-rating experiment (where the correlation coefficients are $-0.08, p>.7$, and $0.39, p>.08$, respectively).

Table 2 shows that many words paired with circle in the consensus measures in Table 1 are rated higher when paired with circle in a metaphor than they are when paired with square. In contrast, only one square word (father) is rated reliably higher when it was paired with square in a metaphor (1.61) rather than with circle (0.67). In only two cases were the mean ratings of circle words lower when they were paired in metaphors with circle rather than with square (mother and odd), whereas more than half of the mean ratings ( 13 cases) of the square words were slightly higher when they were paired with circle rather than with square. However, of the 13 cases, only three were significantly different from chance. Experiment 1 indicated that the properties of a circle lend themselves for use as symbols for the referents being examined here somewhat more than the properties of a square do; correspondingly, a circle is slightly more available for use as a metaphoric vehicle than is a square. But, we emphasize, symbolic use of a circle is rated more favorably than metaphoric use.

The overall low ratings and the frequent absence of clear distinctions between uses of the two forms suggest that the sentences were treated as poor metaphors, unless they are clichés. An apt symbol may not work as a metaphor. A circle may be a good symbol for mother, but "Mother is a real circle" is not an apt metaphor. A circle may be a good symbol for softness. It does not offer a good metaphor for softness.

Although form symbolism is not readily translated into metaphor, could it be related to analogy?

\section{EXPERIMENT 3 Rating the Shapes as Analogies}

If a circle can symbolize a mother and a square can symbolize a father, then likely an analogy can be formed: A circle is to a square as a mother is to a father. An example of an analogy is "an electron is to a nucleus as the earth is to the sun." The analogy is incongruent if terms can be reversed to form "an electron is to a nucleus as the sun is to the earth." In order to test the relation between form symbolism and analogy, two conditions were used as a between-subject factor. In one, called the shape-astopic condition, forms were used as the first, or topic, domain and the word-pairs as the second, or vehicle, domain (e.g., "a circle is to a square as a mother is to a father"). In the other, called the shape-as-vehicle condition, the two were reversed (e.g., "a mother is to a father as a circle is to a square"). Analogies that are congruent and incongruent with the matches shown in Table 1 were also tested.

If form symbolism is related to analogy, then the ratings of the congruent analogies should be correlated with the ratings of the symbols.

\section{Method}

Subjects. Two groups of undergraduate subjects were drawn from an introductory psychology course and were tested individually. There were 24 subjects (ranging in age from 19 to 24 years with a median age of 19,17 of whom were females) in the shape-as-topic group and 21 (ranging from 18 to 22 years of age, with a median age of 19,14 of whom were females) in the shape-as-vehicle group.

Materials and Procedure. Subjects were given sentences to rate as analogies. Subjects were given "An electron is to a nucleus as the earth is to the sun" as an example of an apt analogy. The second page contained sentences in the form " $A$ is to B as C is to D." For the shapeas-topic group of subjects, A and B were the two shapes (circle and square), while $C$ and $D$ were a pair from the list shown in Table 1 . The adjectives in Table 1 were given in noun form. For the shape-as-vehicle group of subjects, the words replace $A$ and $B$ and the shapes $C$ and D. Each subject had two sentence conditions, congruent and incongruent. Each sentence condition contained 20 sentences constructed from the 20 word-pairs.

There were two kinds of orders using congruent sentences and two using incongruent sentences In the shape-as-topic condition, a congruent sentence could either be "a circle is to a square as a mother is to a father" or "a square is to a circle as a father is to a mother." An incongruent sentence could either be "a circle is to a square as a father is to a mother," or "a square is to a circle as a mother is to a father." The same balanced orders were used in the shape-as-vehicle condition. The orders were evenly balanced across subjects.

Each subject received a different randomized order of sentences in the questionnaires. Subjects rated the appropriateness of each analogy on a 0 to 5 point scale.

\section{Results and Discussion}

Mean ratings were higher for congruent than for incongruent analogies in both the shape-as-topic (congruent 2.98, incongruent 2.15) and the shape-as-vehicle (congruent 3.05 , incongruent 2.55$)$ conditions $\left[F(1,43)=28.05, M S_{\mathrm{e}}=\right.$ $0.37, p<.0001]$. No difference was found between shapeas-topic and shape-as-vehicle conditions, and these conditions did not interact with the congruent and incongruent conditions.

A correlation analysis using the mean rating scores and the consensus scores from Table 1 found positive correlations between the levels of consensus and the congruent condition in the shape-as-vehicle analogies $(r=.53, p<$ .02 ), between the levels of consensus and the congruent condition in the shape-as-topic analogies $(r=.79, p<$ .0001 ), and between the congruent shape-as-vehicle analogies and the ratings for the congruent shape-as-topic analogies $(r=.66, p<.001)$. The correlations between the levels of consensus and the incongruent analogies (shape-as-vehicle and shape-as-topic) were nonsignificant.

Interestingly, the ratings for analogies were higher than those for metaphors, even in the case of incongru- 
ent analogies. A discussion with the subjects suggested that at times some subjects ignored the order of the terms in the analogies and, in effect, treated the analogies as if they were in congruent order by asking themselves whether there was an acceptable order for the terms in the analogies. We have found this to be a relatively common error in multiple-choice examinations.

\section{GENERAL DISCUSSION}

Experiments 1 and 3 indicate that the shift from symbolism to analogy does not require a change in the criteria that the topic and vehicle must meet. Experiment 2 indicates that stiffer criteria are demanded by metaphors.

In solving a symbolic-form task, subjects may consider that a pair of terms imply a relevant set: a circle is related to mother if a square is related to father. Very often, the set may be larger than four-term, just as a circle may be understood to be a member of a set of basic Euclidean forms (circle, square, triangle, etc.), the referents may be taken to be of another set (mother, father, child, uncle, aunt, etc.) When subjects are invited to ascertain which form would be a good symbol for a given referent, they may generally consider not only that the vehicle is part of a large set of items, but also that the same relations hold in the sets to which the topic and vehicle belong - that items have opponents, contraries, subordinates, alternates, and other relations readily available to be invoked (Gentner \& Jeziorski, 1993). We stress, however, that the mere existence of a large set of relevant items and possible relations implied by a topic and a vehicle does not mean that the key, relevant relation between the two terms of the statement linking the topic and vehicle is a strong one. Rather, a hundred weak relations may not rival one strong relation, inasmuch as, the more distant the item from the central comparison, the less it may be able to contribute. Only if there is a strong relevant relation may the comparison be available for use as a metaphor.

Although symbolism may be expressible in the form of an analogy, analogy is at times used in explanations. Analogies use the known (orbits of planets) to explain the unknown (motions of electrons around the nucleus). In contrast, we are unlikely to arrive at a way to understand mother via a circle. Quite the reverse: Likely, we accept mother as symbolized by a circle because we already know a set of relevant features of both mother and circle. This is why, we suggest, congruent shape-as-vehicle and shape-as-topic conditions in Experiment 3 provided similar results. The common features are known and do not have to be taken from one particular source where they are familiar and then applied to the other (Liu \& Kennedy, 1996).

Glucksberg and Keysar (1990) suggest that metaphors may involve class-inclusion, using especially prominent instances of a more general type. "Sam is a pig" uses pig as a good example of a general type. Shape symbolism, however, fails to function readily as a metaphor and surely does not involve class-inclusion via especially apt examples. Certainly, mother and happiness are not good instances of circles, just as circles are not instances of mother or happiness. We suggest that it is likely that circles should possess a useful feature in common with their referents in order to be apt novel symbols that produce consensus, but a circle need not be a prime example of the items with that feature. That key feature is only one of many, and it is not especially prominent. To make the feature prominent requires more resources than those supplied by the form of a metaphor. An analogy may be related closely to a symbol because it too can use examples that are not especially striking instances of the category at issue.

The extra resource to make the examples that are acceptable as a form symbol or an analogy become a metaphor is often supplied by a particular cultural group, and the result can become a familiar cliché, we suggest. "He is running around in circles" implies confusion and panic. An old-fashioned person is a square. A personality can be crooked (dishonest), or straight (direct). These metaphorical propositions have extracted one from a form's many, equally prominent features, one not distinguished in salience, and made it relevant for a pe- riod. Circle, in "he is running around in circles," has been disambiguated so that warm and happy are excluded. What have probably been preserved are endless, pointless, and the like. Endless, despite any amount of effort, indicates confusion, being lost, and so forth.

Some metaphors make sense fairly readily on their own, in isolation, on first exposure - for example, "That politician had all the ethics of a young cuckoo." But "warmth is a circle" is obscure. We need a context in order to select several possible bases for meaning. The context can be a matter of significant but essentially random events in a language community. "He lucked out" means the opposite of "he was out of luck"--surely a matter of chance. If chance events at some stage in language change had been otherwise, the sentence "he was running around in circles" could have meant especially happy for English speakers. Differences between "He lights my fire!" and "He burns me up!"- the first indicating attraction and the latter strong annoyanceare likely a matter of chance, capitalizing on a prior tendency to pair emotion with warmth. Forms offer many possible apt referential functions, and language communities can select one or another for a time, largely as a result of chance events.

For running around in circles the disambiguating context was likely additional earthy phrases such as like a chicken with its head cut off. If a cynic had written about joyous people that "they are singing, praising, and running around in circles," then being overjoyed could have become a standard referent of running around in circles, and, with time, this could become watered down to just mean very happy.

Many shapes can function as symbols (Liu, 1997; Liu \& Kennedy, 1993; Marks, 1996; Schlesinger, 1980). Referents of circle and square as symbols are also referents of other forms that share many properties with them-a sphere and a cube, curved and angular (Liu, 1997). Elsewhere we have reported similar results for subjects making visual matches of visible forms (circle and square) and lists of words (soft, warm, etc.). Also, blind persons given the words circle and square in oral presentation and asked to pair them to the list of word-pairs shown in Table 1, produced essentially the same consensus as here (Kennedy, 1997; Liu \& Kennedy, 1993). Hence, as far as these two shapes are concerned, the medium of presentation has been found to be unlikely to produce much change in consensus.

In sum, metaphor may require stronger common properties than do analogy and symbolism.

\section{REFERENCES}

Gentner, D., \& Jeziorski, M. (1993). The shift from metaphor to analogy in Western science. In A. Ortony (Ed.), Metaphor and thought (pp. 447-480). New York: Cambridge University Press.

GlucksberG, S., \& Keysar, B. (1990). Understanding metaphorical comparisons. Psychological Review, 97, 3-18.

Gomвrich, E. H. (1963). Visual metaphors of value in art. In E. H Gombrich (Ed.), Meditations on a hobby horse (pp. 12-29). London: Phaidon.

GomBrich, E. H. (1965). The use of art for the study of symbols American Psychologist, 20, 34-50.

HAUSMAN, C. R. (1989). Metaphor and art: Interactionism and reference in the verbal and nonverbal art. New York: Cambridge University Press

IndURHKYA, B. (1992). Metaphor and cognition. Cambridge, MA: MIT Press.

JoHnson, M. (1991). The body in the mind: The bodily basis of meaning, imagination and reason. Chicago: University of Chicago Press

Kennedy, J. M. (1990). Metaphor: Its intellectual basis. Metaphor \& Symbolic Activity, 5, 1 15-123.

Kennedy, J. M. (1993). Drawing and the blind: Pictures to touch. New Haven, CT: Yale University Press.

Kennedy, J. M. (1997, January). How the blind draw. Scientific American, 276, 76-81.

Kennedy, J. M., Green, C. D., \& Vervaeke, J. (1993). Metaphoric thought and devices in pictures. Metaphor \& Symbolic Activity, 8 , 243-255.

LEACH, E. (1976). Culture and communication: The logic by which symbols are connected. Cambridge: Cambridge University Press. 
LiU, C. H. (1997). Symbols: Circles and spheres represent the same referents. Metaphor \& Symbol, 12, 135-147.

LiU, C. H., \& KenNEDY, J. M. (1993). Symbolic forms and cognition. Psyke \& Logos, 14, 441-456.

LiU, C. H., \& KenNedy, J. M. (1994). Symbolic forms can be mnemonics for recall. Psychonomic Bulletin \& Review, 1, 494-498.

LiU, C. H., \& KENNEDY, J. M. (1996). Form and its symbolic meaning. In R. Woodfield (Ed.), Gombrich on art and psychology (pp. 127 139). Manchester, U.K.: Manchester University Press.

MarKS, L. E. (1996). On perceptual metaphors. Metaphor \& Symbolic Activity, 11, 39-66.
RICHARDS, I. A. (1936). The philosophy of rhetoric. Oxford: Oxford University Press.

SCHLEsinger, L. B. (1980). Physiognomic perception: Empirical and theoretical perspectives. Genetic Psychology Monographs, 101, 71-97. Tourangeau, R., \& Sternberg, R. J. (1981). Aptness in metaphor. Cognitive Psychology, 13, 27-55.

Tourangeau, R., \& SternberG, R. J. (1982). Understanding and appreciating metaphors. Cognition, 11, 203-244.

(Manuscript received September 3, 1996; revision accepted for publication June 3, 1997.) 